\title{
Initial Development of a Master-Slave Controller for a Five-Fingered Robotic Hand Design by Using Pressure Sensors Comparator Technique
}

\author{
Syed Zainal Abidin Syed Kamarul Bahrin ${ }^{1}$, Khairul Salleh Mohamed Sahari², \\ ${ }^{1}$ Department of Electrical Power Engineering, Universiti Tenaga Nasional \\ ${ }^{2}$ Department of Mechanical Engineering, Universiti Tenaga Nasional \\ *Corresponding author E-mail: SZainal@uniten.edu.my
}

\begin{abstract}
There are numerous robotic hand designs but the five-fingered robotic hand design is the most dexterous robotic hand design due to its similar appearance and motions with the human hands. The five-fingered robotic hands are commonly controlled or governed through a master-slave system that can be accomplished by using simple preset motions or other complicated and advanced technologies. However, a five-fingered robotics hand can also be controlled by a novel approach known as pressure sensors comparator technique. This technique compares the values from the pressure sensors that are strategically located at the glove (master) and robotic hand (slave). If the values differ, the actuators will generate motions accordingly. The initial finding based on the master and slave prototypes showed that applying this technique is very challenging due to the humans' physiological diversity. Nevertheless, a solution was proposed for further studies and future developments by introducing an offset.
\end{abstract}

Keywords: robotics; robotic hand; five-fingered robotic hand; master-slave; pressure sensor; flex sensor; bend sensor.

\section{Introduction}

\subsection{Overview}

Robotics is a technology that consists of processes or actions that are involved in designing, constructing, operating and developing robots [1]. The technology also includes the computer systems for controlling, sensory feedback and information processing of robots. These technologies are very important in performing a wide range of tasks that are very difficult or impossible to be executed solely by humans due to various hazardous elements or safety issues such as performing dangerous manufacturing processes, handling biohazard elements, disarming bombs, post-disaster search and rescue missions, etc. There are several robotic components involved to perform these tasks but the most utilised component is the robot's hand because it has more degrees of freedom (DOF) compared to the other components.

Robotic hand is available in a wide range of designs but the fivefingered robotic hand design has the highest number of DOF. This is because a five-fingered robotic hand is designed to be more dexterous compared to the other robotic hand designs as it imitates a human hand in term of appearances and motions.

A five-fingered robotic hand can be controlled through various methods but the master-slave controller scheme is considered to be the most widely used system. In a master-slave system, the user can control the robotic hand by using microcontrollers with preset motion settings [2] [3], visual monitoring [4] [5], haptic feedback [5] [7] and CyberGlove [8]-[10]. However, the robotics hand can also be controlled by a novel approach known as pressure sensors comparator technique. The pressure sensors comparator technique utilises microcontrollers to compare the values of pressure at each joints of the robotic hand fingers with the values of pressure at each joints of a specially designed glove. If the pressure sensor's value at one joints of the robotic hand finger is not the same as the pressure sensor's value at the similar joints of the glove, the microcontroller will activate the actuators to move the finger. This comparing technique allows the robotic hand to move rigorously according to the glove motions as long as the pressure sensors have similar characteristics.

\subsection{Pressure Sensor Applications in Robotics}

Pressure sensors are widely used for various domestic, industrial, agricultural, manufacturing and even military applications. Thus, pressure sensors are also widely used in robotics applications due to its importance and existence in each sector. However, pressure sensors were never used to regulate motions between the glove and robotic fingers meticulously. This is because these sensors are typically used for angle positioning and tactile sensing that are placed at strategic locations.

\subsubsection{Angle Positioning}

The types of pressure sensors that are usually used for positioning are flex sensor and bend sensor. These sensors have similar fundamental concept; the resistant of the sensors will increase as the flexing or bending angle increases. The changes are linearly proportional but the resistance at $0^{\circ}$ angle has a tolerance value of $\pm 30 \%$, which is quite high and not suitable for sensitive or precision applications when the sensor is at relaxed position.

The sensors can be integrated with a glove or robotic fingers to perform various tasks by indentifying the flexing or bending angle. If the sensors are integrated with a glove, the output angles 
can be used to translate gestures [11] [12] or control the actuators to move the fingers according to the glove's motions [13] - [15]. The later application is generally used in rehabilitation for hand impaired patients. The sensors can also placed on the skin directly based on the recent development [16] but this type of sensor has lower sensitivity and higher relaxation settling time as compared to flex and bends sensors [17]. In the aspect of integrated sensors within robotic fingers, the main function is for validity test to ensure the motions are within the expected range [18] [19].

\subsubsection{Tactile Sensing}

Tactile sensing is an action that processes information from physical interaction with the surrounding environment through the biological sense of touching. Tactile sensor is a type of pressure sensor that is either made from piezoresistive, piezoelectric, capacitive and elastoresistive materials. The sensors are usually arranged in arrays to achieve optimum sensing.

The sensors are normally used in robotics to prevent assist robot from injuring the user [20], controlling the grasping strength of a robotic hand or fingers [21] - [27], obstacle detection [28], integration with haptic devices to provide feedback to the user [29] [30], thin sheet manipulation [31] and object recognition/identification [32]. Based on these applications, it can be concluded that the main function of the sensor is to prevent failures and damages to the handled objects and the robotic hand itself.

\subsection{Pressure Sensors Comparator Technique}

As stated before, pressure sensors were never used to strictly regulate motions between the glove and robotic fingers because these sensors are usually used for positioning and sensing. Therefore, this paper will firstly introduce the pressure sensor comparator technique by looking at the master and slave components, and the proposed comparator system. The prototype master and slave sensors' resistances will then be measured to determine the actual outputs when both components are making similar finger's action.

\section{Master and Slave Designs}

The glove and robotic hand are designed in accordance to a master and slave relationship respectively by using flex sensors (a pressure based sensor) and the solenoid actuated five-fingered robotic hand design (developed by the authors) as reference [33]. This particular relationship will force the robotic hand to strictly adhere to the glove's movements. Therefore, some duplicated structural designs were introduced to ensure proper instructional processing and executions from both parts.

\subsection{Master Components}

The master components design is shown in Figure 1, Figure 2 and Figure 3 for the top, base and perspective views respectively. It can be seen that the master or glove attachment design is partially based on the top part of a solenoid actuated five-fingered robotic hand's fingers and backhand. This design is modular and can be attached to any commercially available or handmade gloves. It is not shown clearly in the figures but it is important to highlight that the phalanges and backhand components are not connected to one another. These components can be freely arranged and attached to an existing glove for better flexibility and optimised sensors' positioning.

\subsection{Slave Components}

The slave components design is shown in Figure 4, Figure 5 and Figure 6 for the palm, backhand and perspective views respectively. It is important to highlight that the slave or hand design is the actual solenoid actuated five-fingered robotic hand design.
This similar base design selection is important to reduce deviations in the pressure sensor's readings that might occur when the finger moves due to inconsistent structural design. There is always a possibility that the pressure sensor will bend inappropriately when the structural design is not consistent.

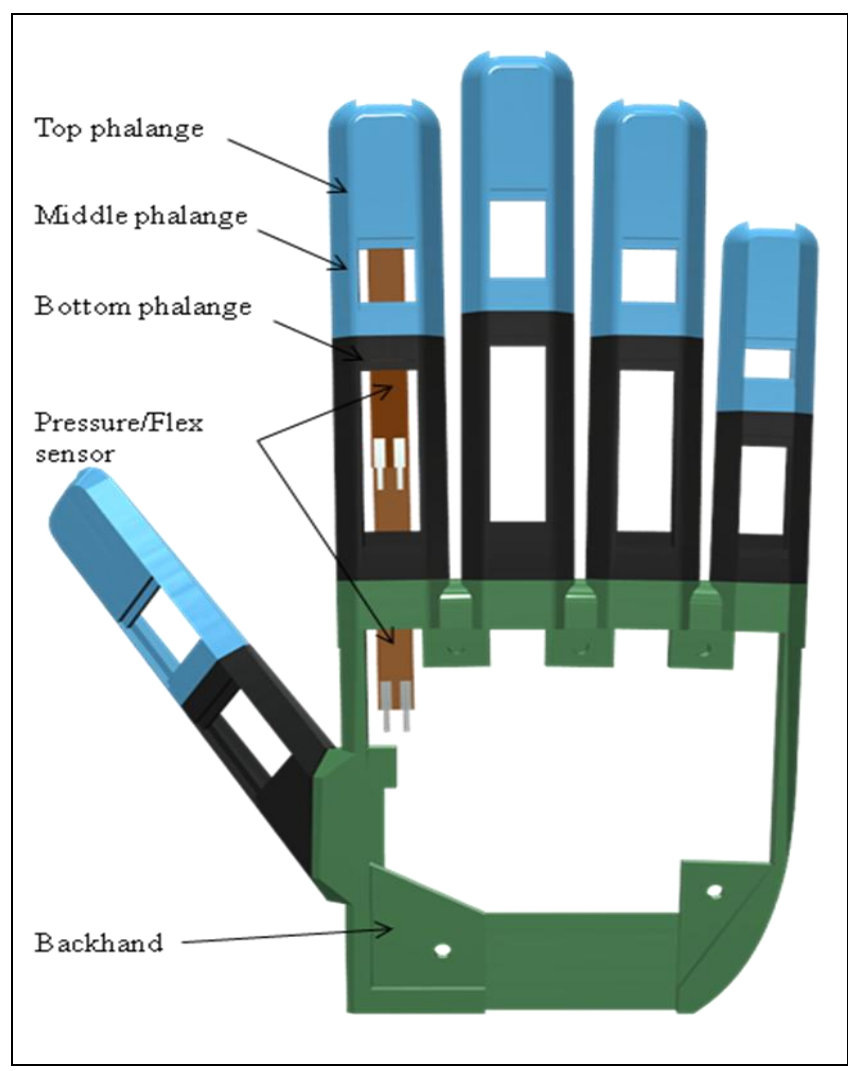

Fig. 1: Top view of the master components or glove design.

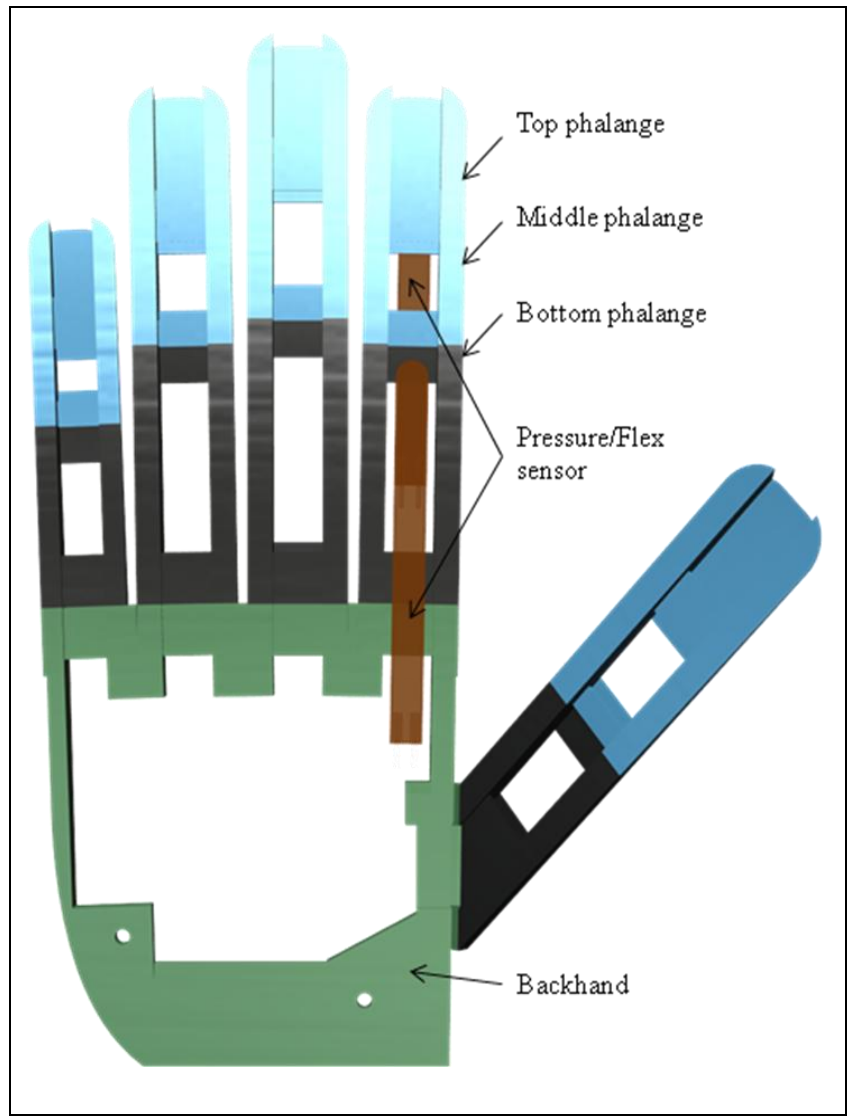

Fig. 2: Base view of the master components or glove design. 


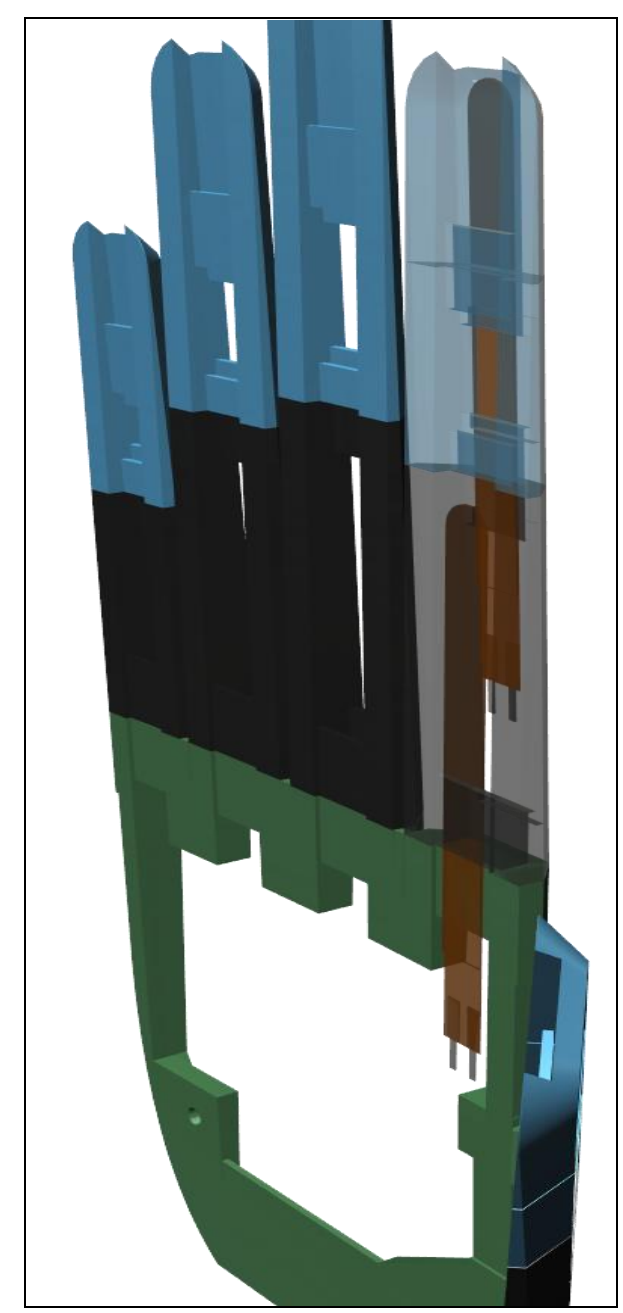

Fig. 3: Perspective view of the master components or glove design to emphasise the sensors placement.

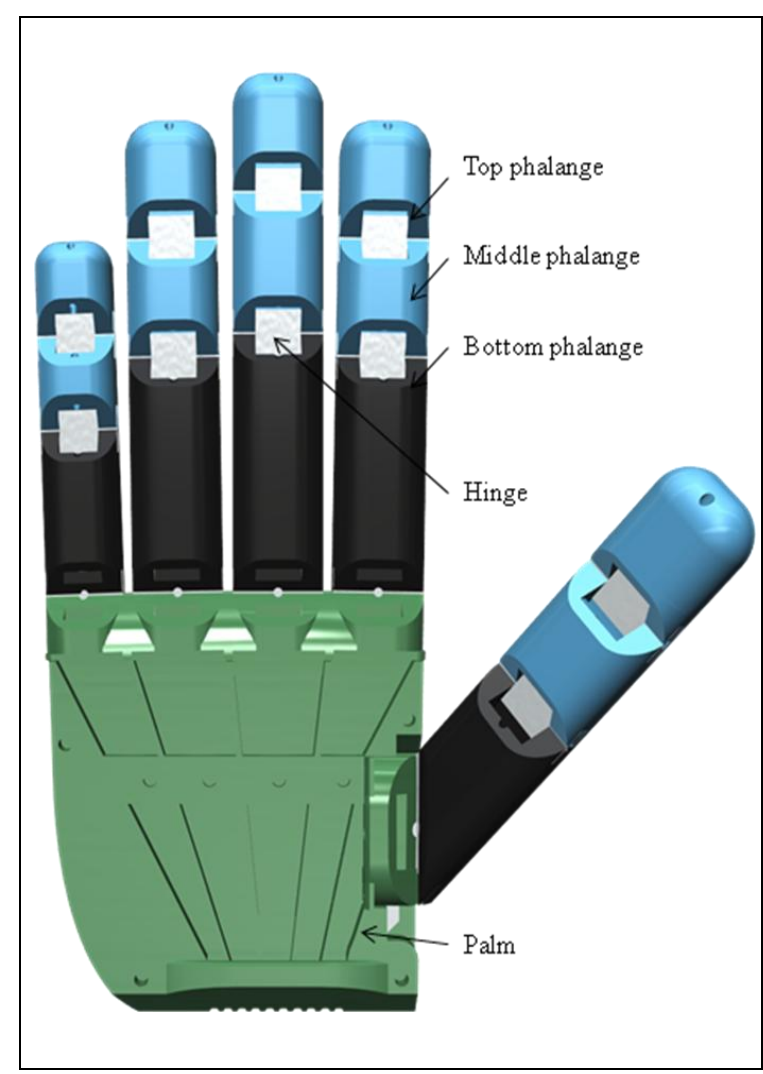

Fig. 4: Palm view of the slave components or hand design.

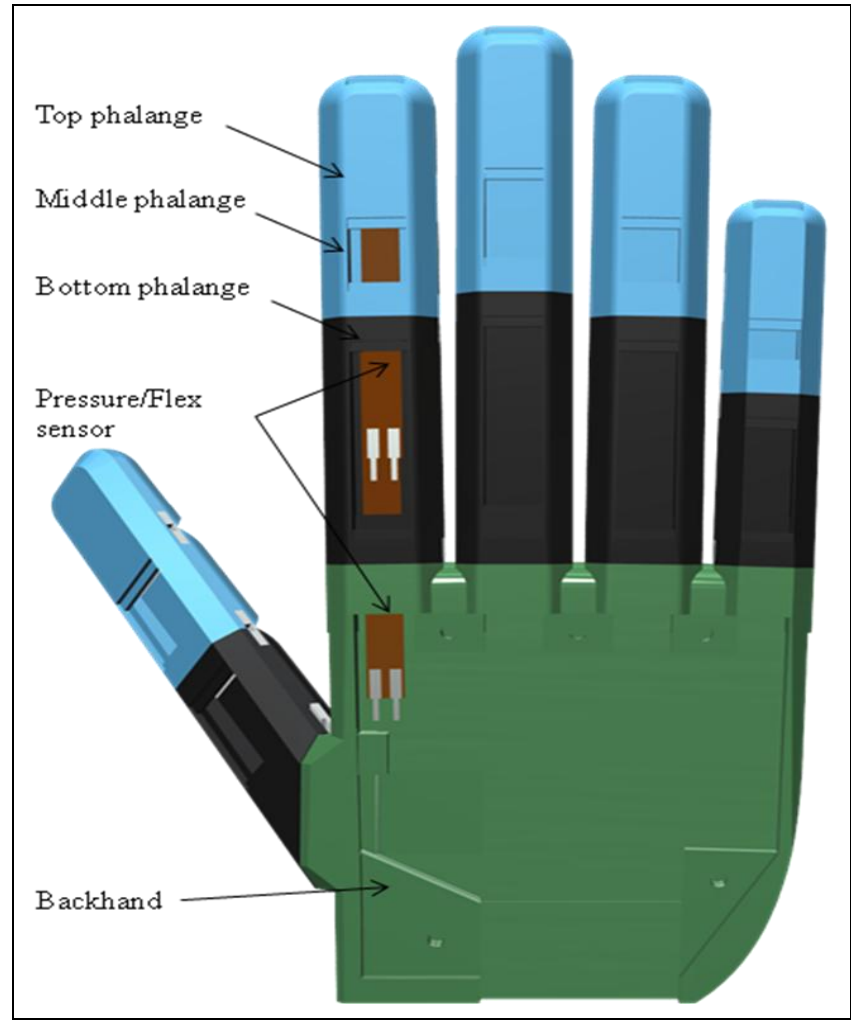

Fig. 5: Backhand view of the slave components or hand design.

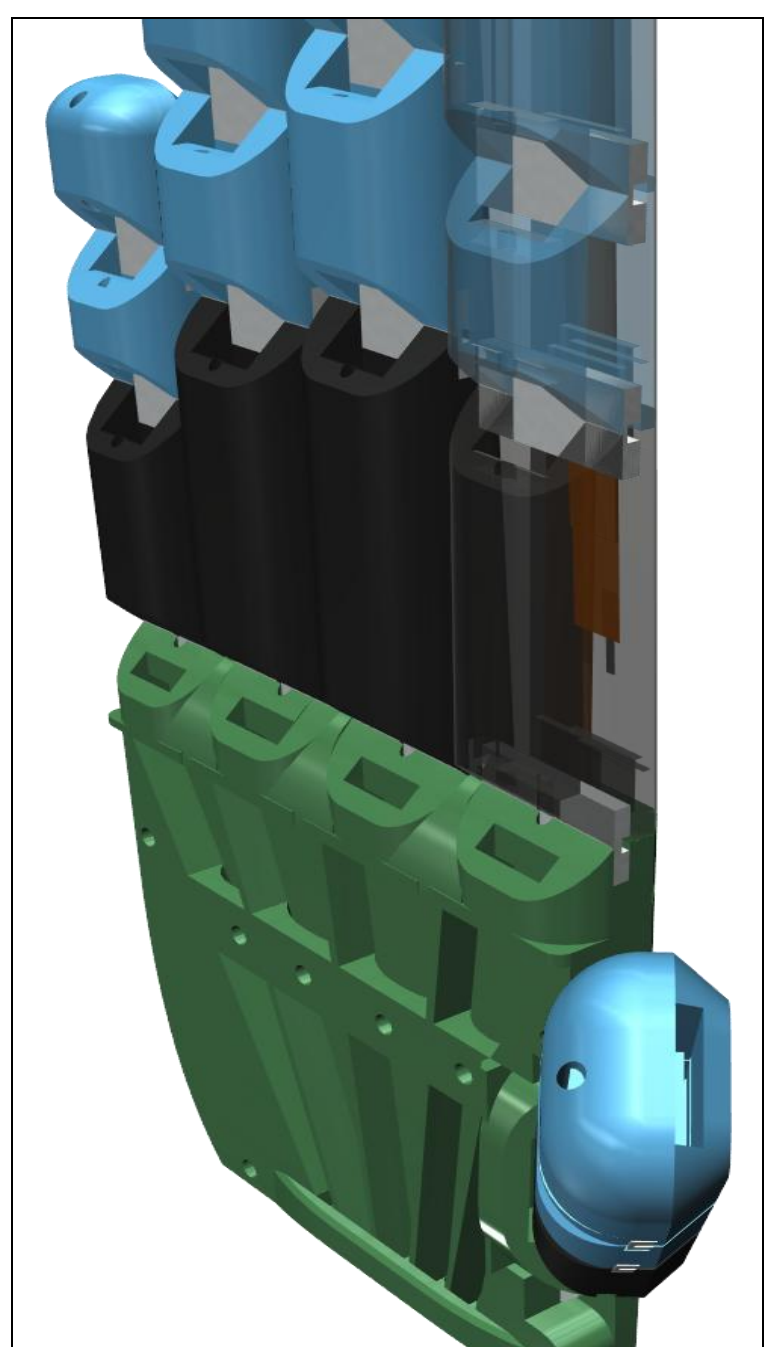

Fig. 6: Perspective view of the slave components or hand design to emphasise the sensors placement. 


\section{Pressure Sensors Comparator System}

The pressure sensors comparator system, which is shown in Figure 7, is based on a closed loop system that regulates the actuators' movements by comparing the master and slave sensors' values. Essentially, the actuator for one finger will move as long as the sensor reading from a specific finger at the master is not similar with the sensor reading from a specific finger at the slave. It is important to note that the specified finger from both components must be similar i.e. index finger with index finger.

\begin{tabular}{|c|c|c|}
\hline Glove Sensor & $\rightarrow$ Microcontroller & ive \\
\hline & $\begin{array}{c}\text { Robotic Hand } \\
\text { Sensor }\end{array}$ & \\
\hline
\end{tabular}

Fig. 7: The pressure sensors comparator system.

The recursive process can be clearly explained by using one finger and one pressure sensor, each from the master and slave as example. Initially, the microcontroller will read the values from the master and slave sensors. The value for each sensor will vary when the bending or flexing angle of the sensor varies because the sensor's resistance will increase linearly as the bending or flexing angle increases. Then, the microcontroller will use the values to compute an output to the drive through a control function (will not be discussed in this paper) that consists of calculations and logic functions. The drive will set a suitable voltage at the actuator that causes the finger to move into curling position (Figure 8 (a)) when the master sensor's value is larger than the slave sensor's value or straighten position (Figure 8 (b)) when the slave sensor's value is larger than the master sensor's value. Since the slave sensor's value will also change when the actuator moves due to the sensor's location within the finger, the actuator will keep on moving until both sensors' values are similar. This recursive process will cause the robotic hand to follow the glove's movements. A flowchart is shown in Figure 9 to provide a simple illustration on this recursive process.

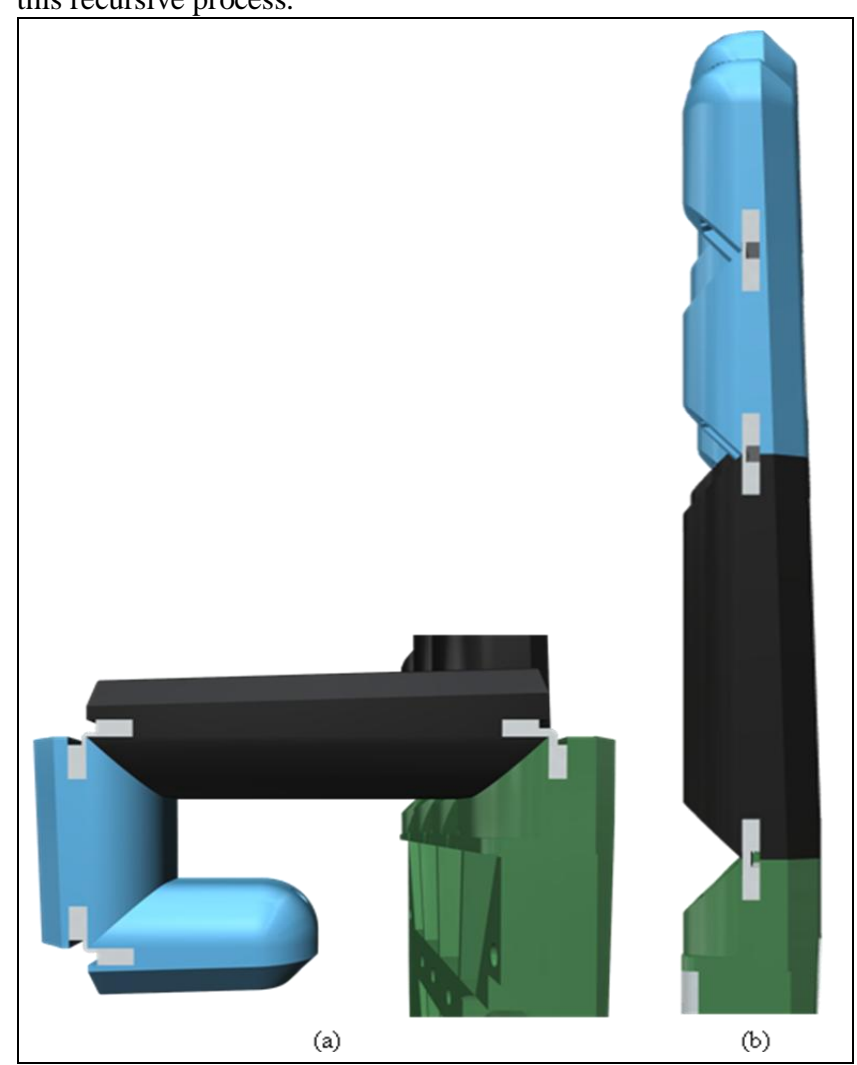

Fig. 8: Robotic hand in (a) curling position and (b) straighten position.

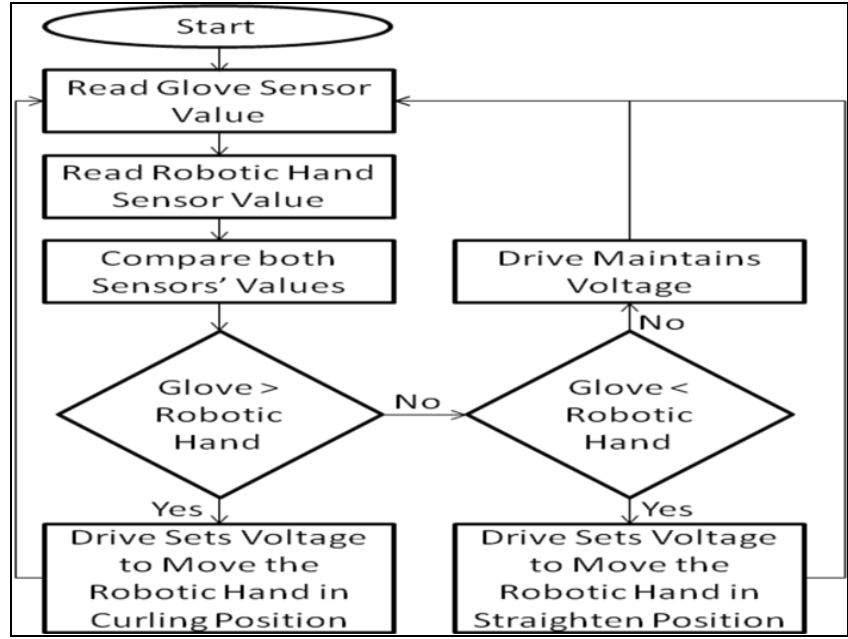

Fig. 9: Pressure sensors comparator system flowchart.

\section{Master and Slave Sensors Readings}

The pressure sensors used for the master and slave are similar but the values vary by $\pm 30 \%$ due to the manufacturing tolerance based on the datasheet. Therefore, it is important to measure the pressure sensors from the actual master and slave prototypes in the initial development to determine whether the readings from both components vary greatly when making similar actions. Both prototypes were $3 \mathrm{D}$ printed based on the design in Section 2 to ensure consistency when the finger motions and resistance values of the pressure sensors are compared.

\subsection{Master Prototype}

The master prototype is shown in Figure 10. It is important to note that the angle between the fingers is now slightly larger as compared to the original design due the glove's design. Even though the modular design allows flexibility in placing the master components, the pressure sensors might be shifted from its original position when the finger moves because the human's backhand is not flat. These particular shifting actions might prevent the pressure sensors from bending in one direction.

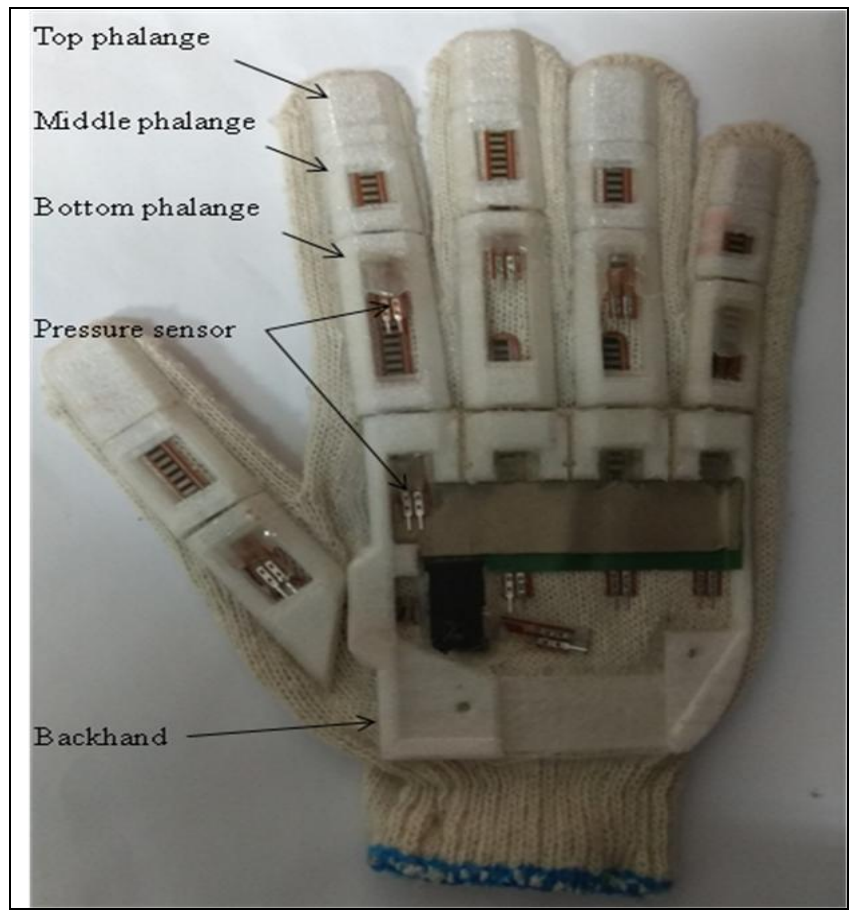

Fig. 10: Master prototype components. 


\subsection{Slave Prototype}

The master prototype is shown in Figure 11, Figure 12 and Figure 13 for the palm, backhand and side views respectively. Notice that the master component is similar to the slave's backhand shape.

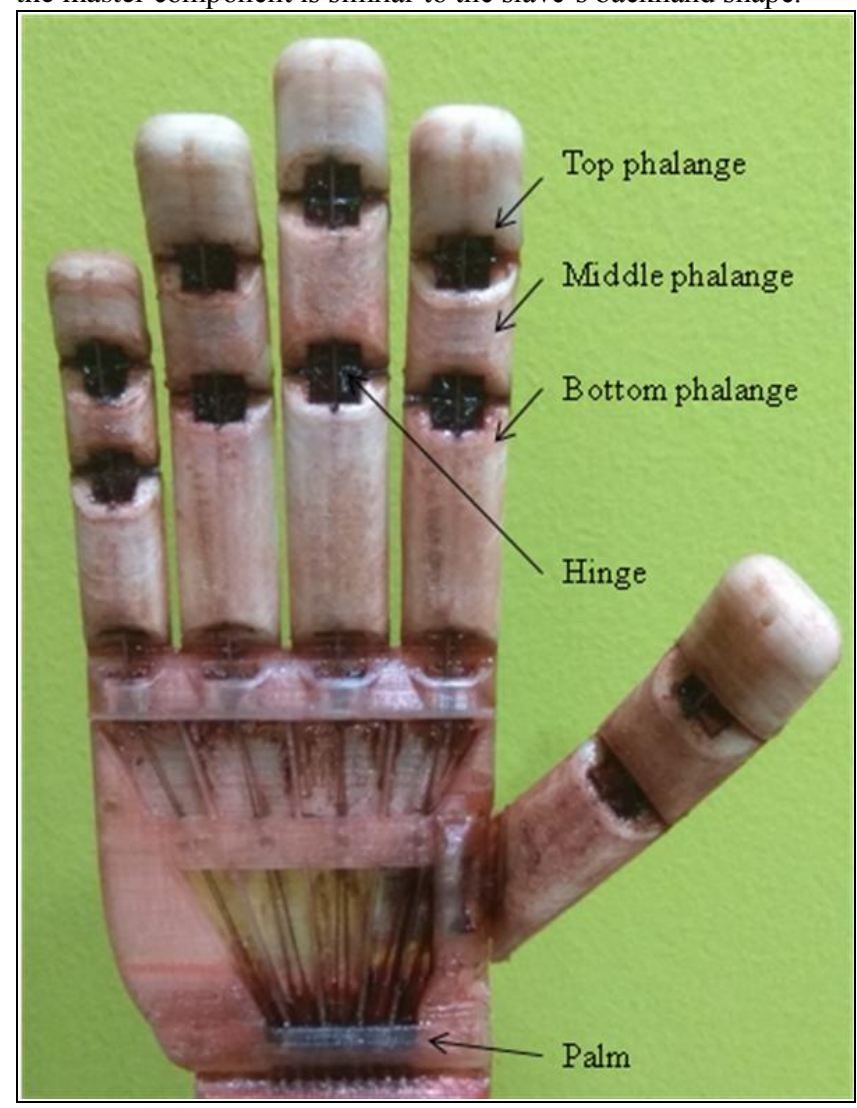

Fig. 11: Slave prototype palm view.

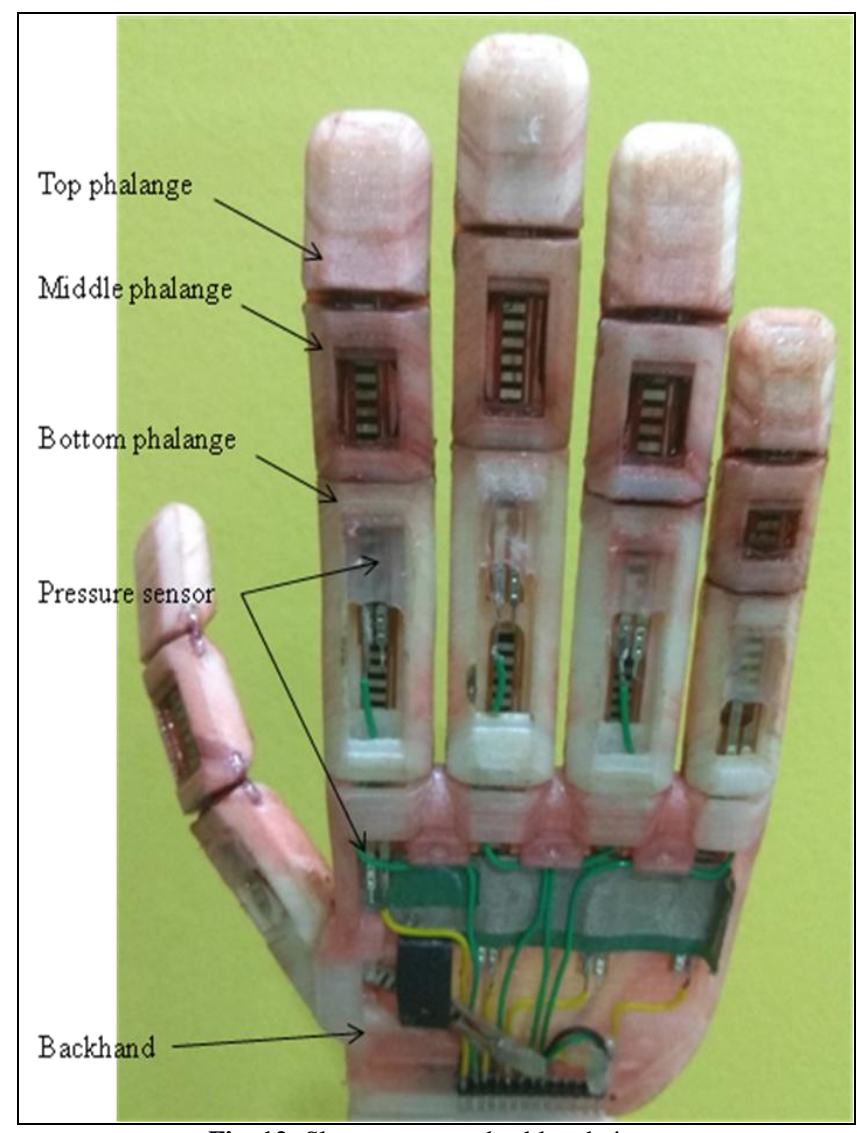

Fig. 12: Slave prototype backhand view.

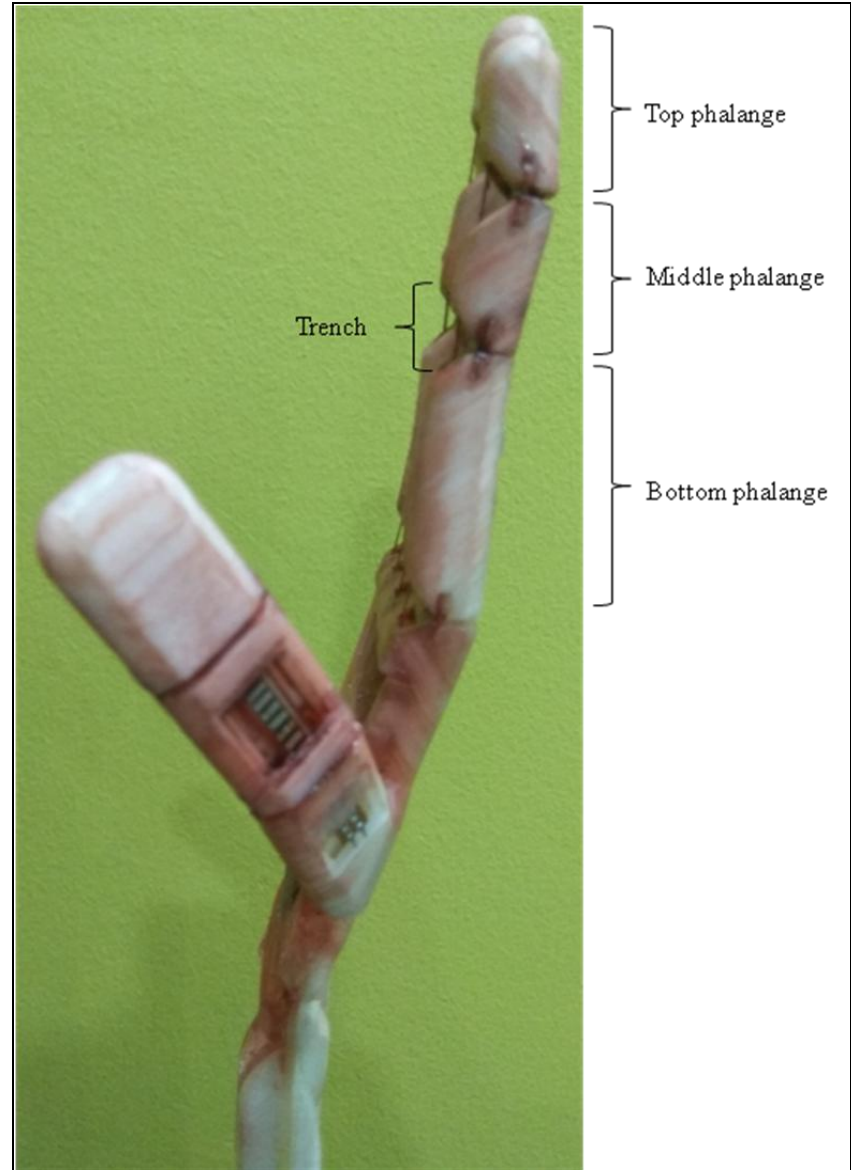

Fig. 13: Slave prototype side view.

\subsection{Sensors Reading}

Figure 14 illustrates the index fingers' angular position for both master and slave prototypes.

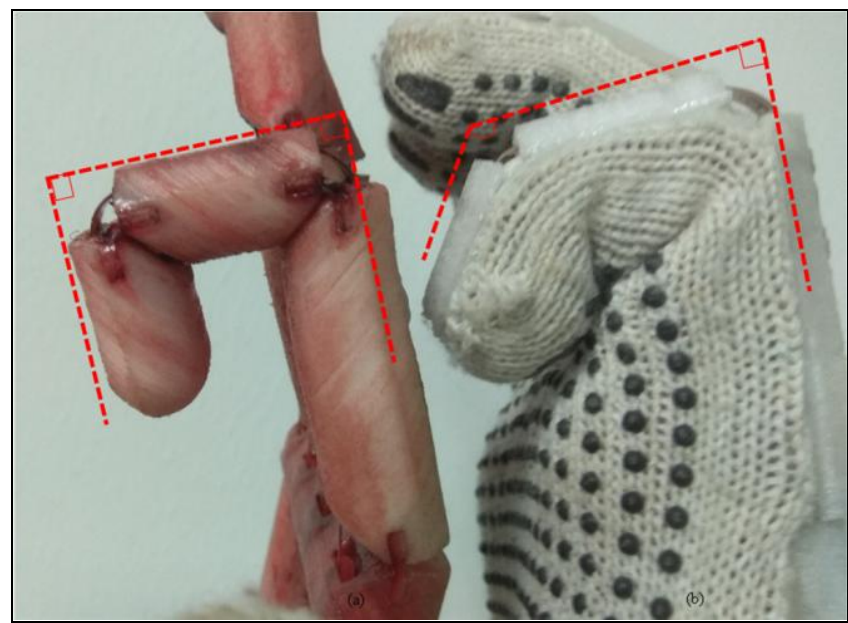

Fig. 14: The (a) slave prototype and (b) master prototype demonstrate a hooking gesture through the index finger. The red dashes lines indicate the angular positions.

Based on Figure 14, it can be seen that the angular position for the slave prototype and master prototype is not the same when both prototypes are making a hooking gesture. The slave component can make $90^{\circ}$ bending between top-middle phalange and middlebottom phalange but the master component can only make a $90^{\circ}$ bending between mid-bottom phalange only. Since the angular position is not similar, the resistance values of the pressure sensors are not identical or close to each other too. The readings showed that the master's pressure sensor outputs a value of $70 \mathrm{k} \Omega$ whereas 
the slave's pressure sensor outputs a value of $121.6 \mathrm{k} \Omega$ (bending angle and resistance is directly proportional). This particular problem cannot be avoided because each human has different physiological conditions or appearances. Therefore, an offset should be introduced to solve the sensor's tolerance value and the angular variation between the master and slave problem.

One possible method is to normalise the sensor's value at the master or slave component by deducting the higher value with the lower value to get the offset when the finger is in straighten position. From this point onwards, the higher value readings will be deducted by the offset. For example, if the current master sensor's value is $20 \mathrm{k} \Omega$ and has a higher sensor's value than the slave component during straighten position with a calculated offset of $10 \mathrm{k} \Omega$, then the actual value is $10 \mathrm{k} \Omega$. This method can only be applied if the sensor changes linearly most of the time and this method must obtain the offset every time the microcontroller reboots. Another possible method of offset is to use variable resistors that can set the output readings for the master and slave to be similar when the microcontroller read the values at straighten or curling position. The variable resistors will also act as a memory for the offset calibration every time the power to the microcontroller is turned off.

\section{Conclusion}

Designing a master-slave controller for a five-fingered robotic hand design by using pressure sensors comparator method is possible but there are a lot of inconsistencies because the sensor's tolerance varies individually and the human's hand and fingers appeared in various physiological forms. However, the development is still in its early stage and introducing an offset to the technique or system might solve the problem. Therefore, further studies are required for further developments.

\section{Acknowledgement}

The authors would like to thank the Malaysian government for funding this project in 2013 until 2016 under the Exploratory Research Grant Scheme (ERGS): 06012013ERGS.

\section{References}

[1] "robotics". Oxford Dictionaries. Retrieved 20 February 2013.

[2] Cabas, R.; Balaguer, C.; , "Design and development of a light weight embodied robotic hand activated with only one actuator," Intelligent Robots and Systems, 2005. (IROS 2005). 2005 IEEE/RSJ International Conference on , vol., no., pp. 2369- 2374, 2-6 Aug. 2005

[3] Hyunhwan Jeong; Joono Cheong; , "Design of hybrid type robotic hand : The KU hybrid HAND," Control, Automation and Systems (ICCAS), 2011 11th International Conference on , vol., no., pp.1113-1116, 26-29 Oct. 2011.

[4] Lippiello, V.; Ruggiero, F.; Siciliano, B.; Villani, L.; , "Visual Grasp Planning for Unknown Objects Using a Multifingered Robotic Hand," Mechatronics, IEEE/ASME Transactions on , vol.18, no.3, pp.1050-1059, June 2013.

[5] Khalil, F.F.; Curtis, P.; Payeur, P.; , "Visual monitoring of surface deformations on objects manipulated with a robotic hand," Robotic and Sensors Environments (ROSE), 2010 IEEE International Workshop on, vol., no., pp.1-6, 15-16 Oct. 2010.

[6] Yoshimura, Y.; Ozawa, R.; , "A supervisory control system for a multi-fingered robotic hand using datagloves and a haptic device," Intelligent Robots and Systems (IROS), 2012 IEEE/RSJ International Conference on, vol., no., pp.5414-5419, 7-12 Oct 2012.

[7] Panarese, A.; Edin, B.B.; Vecchi, F.; Carrozza, M.C.; Johansson, R.S.; , "Humans Can Integrate Force Feedback to Toes in Their Sensorimotor Control of a Robotic Hand," Neural Systems and Rehabilitation Engineering, IEEE Transactions on , vol.17, no.6, pp.560-567, Dec. 2009.
[8] Karnati, N.; Kent, B.A.; Engeberg, E.D.; , "Bioinspired Sinusoidal Finger Joint Synergies for a Dexterous

Robotic Hand to Screw and Unscrew Objects With Different Diameters," Mechatronics, IEEE/ASME Transactions on, vol.18, no.2, pp.612-623, April 2012.

[9] Jie Liu; Yuru Zhang;, "Mapping human hand motion to dexterous robotic hand," Robotics and Biomimetics, 2007.ROBIO 2007. IEEE International Conference on , vol., no., pp.829-834, 15-18 Dec. 2007.

[10] Backdrivable Periodic Finger Joint Synergies: Human Observations Applied to a Dexterous Robotic Hand Karnati, N.; Kent, B.; Engeberg, E.D.; , "Backdrivable periodic finger joint synergies: Human observations applied to a dexterous robotic hand," Robotics and Biomimetics (ROBIO), 2011 IEEE International Conference on vol., no., pp.1122-1127, 7-11 Dec. 2011.

[11] C. Preetham, G. Ramakrishnan, S. Kumar, A. Tamse and N. Krishnapura, "Hand Talk-Implementation of a Gesture Recognizing Glove," India Educators' Conference (TIIEC), 2013 Texas Instruments, Bangalore, 2013, pp. 328-331.

[12] M. B. H. Flores, C. M. B. Siloy, C. Oppus and L. Agustin, "Useroriented finger-gesture glove controller with hand movement virtualization using flex sensors and a digital accelerometer," Humanoid, Nanotechnology, Information Technology, Communication and Control, Environment and Management (HNICEM), 2014 International Conference on, Palawan, 2014, pp. $1-4$.

[13] A. M. ALmassri, M. B. Abuitbel, W. Z. WanHasan, S. A. Ahmad and A. H. Sabry, "Real-time control for robotic hand application based on pressure sensor measurement," Robotics and Manufacturing Automation (ROMA), 2014 IEEE International Symposium on, Kuala Lumpur, 2014, pp. 80-85.

[14] C. Karaçizmeli, G. Çakır and D. Tükel, "Robotic hand project," 2014 22nd Signal Processing and Communications Applications Conference (SIU), Trabzon, 2014, pp. 473-476.

[15] S. W. Pu, S. Y. Tsai and J. Y. Chang, "Design and development of the wearable hand exoskeleton system for rehabilitation of hand impaired patients," 2014 IEEE International Conference on Automation Science and Engineering (CASE), Taipei, 2014, pp. 996-1001.

[16] T. Someya, "Building bionic skin," in IEEE Spectrum, vol. 50, no. 9, pp. 50-56, September 2013.

[17] Dong Hyun Kim, S. W. Lee and Hyung-Soon Park, "Sensor evaluation for soft robotic hand rehabilitation devices," 2016 6th IEEE International Conference on Biomedical Robotics and Biomechatronics (BioRob), Singapore, 2016, pp. 1220-1223.

[18] A. F. C. Silva, C. da Rocha Souto, S. A. da Silva, A. J. V. dos Santos and E. A. T. Filho, "Application of artificial vision as measurement validation tests on a robotic hand driven by shapememory alloys," 2015 IEEE International Instrumentation and Measurement Technology Conference (I2MTC) Proceedings, Pisa, 2015, pp. 522-526.

[19] J. Morrow et al., "Improving Soft Pneumatic Actuator fingers through integration of soft sensors, position and force control, and rigid fingernails," 2016 IEEE International Conference on Robotics and Automation (ICRA), Stockholm, 2016, pp.

[20] N. Uchiyama, Y. Funabora, S. Doki and K. Doki, "Impedance control based on pressure distribution for wearable assist robot on multi-joint body part," 2015 IEEE International Conference on Control System, Computing and Engineering (ICCSCE), Penang, 2015, pp. 48-53.

[21] J. H. Low et al., "A compliant modular robotic hand with fabric force sensor for multiple versatile grasping modes," 2016 6th IEEE International Conference on Biomedical Robotics and Biomechatronics (BioRob), Singapore, 2016, pp. 1230-1235.

[22] A. M. M. Almassri, W. Z. W. Hasan, S. A. Ahmad and A. J. Ishak, "A sensitivity study of piezoresistive pressure sensor for robotic hand," Micro and Nanoelectronics (RSM), 2013 IEEE Regional Symposium on, Langkawi, 2013, pp. 394-397.

[23] A. A. S. Al-Shanoon, S. A. Ahmad and M. K. B. Hassan, "Investigation of piezoresistive sensor for robotic gripping operations," 2015 IEEE Student Symposium in Biomedical Engineering \& Sciences (ISSBES), Shah Alam, 2015, pp. 54-58.

[24] F. L. Hammond, Y. Mengüç and R. J. Wood, "Toward a modular soft sensor-embedded glove for human hand motion and tactile pressure measurement," 2014 IEEE/RSJ International Conference on Intelligent Robots and Systems, Chicago, IL, 2014, pp. 40004007. 
[25] A. M. ALmassri, W. Z. WanHasan, S. A. Ahmad, A. J. Ishak and C. Wada, "Optimisation of grasping object based on pressure sensor measurement for robotic hand gripper," 2015 9th International Conference on Sensing Technology (ICST), Auckland, 2015, pp. 795-798.

[26] A. A. S. Al-Shanoon, S. A. Ahmad and M. K. B. Hassan, "Regripping analysis based on implementation of slip-detection device for robotic hand model," 2016 IEEE Region 10 Symposium (TENSYMP), Bali, 2016, pp. 203-206.

[27] Y. Tenzer, L. P. Jentoft and R. D. Howe, "The Feel of MEMS Barometers: Inexpensive and Easily Customized Tactile Array Sensors," in IEEE Robotics \& Automation Magazine, vol. 21, no. 3 pp. 89-95, Sept. 2014

[28] A. P. Gerratt, N. Sommer, S. P. Lacour and A. Billard, "Stretchable capacitive tactile skin on humanoid robot fingers - First experiments and results," 2014 IEEE-RAS International Conference on Humanoid Robots, Madrid, 2014, pp. 238-245.

[29] Y. Wang and W. Zhang, "Data glove control of robot hand with force telepresence," 2015 IEEE International Conference on Robotics and Biomimetics (ROBIO), Zhuhai, 2015, pp. 314-319.

[30] R. M. Pierce, E. A. Fedalei and K. J. Kuchenbecker, "A wearable device for controlling a robot gripper with fingertip contact, pressure, vibrotactile, and grip force feedback," 2014 IEEE Haptics Symposium (HAPTICS), Houston, TX, 2014, pp. 19-25.

[31] Y. Fujihira, T. Nishimura and T. Watanabe, "Bent Sheet Grasping Stability for Sheet Manipulation," in IEEE Robotics and Automation Letters, vol. 1, no. 1, pp. 415-422, Jan. 2016.

[32] A. J. Spiers, M. V. Liarokapis, B. Calli and A. M. Dollar, "SingleGrasp Object Classification and Feature Extraction with Simple Robot Hands and Tactile Sensors," in IEEE Transactions on Haptics, vol. 9, no. 2, pp. 207-220, April-June 12016.

[33] S. Z. A. S. K. Bahrin and K. S. M. Sahari, "Design and development of a five-fingered master-slave robotic hand by using solenoid and pressure sensors comparator technology solenoid actuation system," 2016 IEEE International Conference on Power and Energy (PECon), Melaka, 2016, pp. 495-499. 